\title{
O COACHING COMO ESTRATÉGIA DE AUMENTO DO DESEMPENHO DO CAPITAL HUMANO NAS ORGANIZAÇÕES
}

\section{COACHING AS A STRATEGY FOR INCREASING THE PERFORMANCE OF HUMAN CAPITAL IN ORGANIZATIONS}

\author{
Jenifer Lucas Gonçalves- jeniferlucas53@gmail.com \\ Guilherme Augusto Malagolli- guilherne.malagolli@fatectq.edu.br \\ Faculdade de Tecnologia de Taquaritinga (FATEC) - SP - Brasil
}

DOI: 10.31510/infa.v15i2.490

\section{RESUMO}

A busca por inovações tecnológicas está frequentemente associada ao aumento de eficiência de máquinas e equipamentos, no entanto raramente o capital humano das organizações é visto como fonte de inovação na mesma proporção do capital físico. Mesmo assim, a pressão por atingir metas e resultados é uma constante no cotidiano dos funcionários de uma empresa. Neste contexto, o Coaching se coloca como uma prática que busca desbloquear o potencial das pessoas para aumentar o seu desempenho, este artigo tem por objetivo principal analisar a prática com Coaching como fator de aumento de competitividade nas organizações. Como objetivo secundário, busca-se identificar a aceitação do Coaching como método reconhecido e eficaz por parte das empresas. Para isso, a metodologia do artigo está pautada em uma pesquisa qualitativa, de caráter exploratório e analítico, que buscou através de entrevistas com profissionais do Coaching e com empresários para atingir os objetivos propostos. Como resultado, notou-se que o Coaching aborda de forma eficaz temas que frequentemente são causadores de stress no dia a dia das organizações e, assim, causadores de queda de desempenho. Porém, a prática do Coaching ainda é vista como uma novidade por parte dos empresários, que apresentam certa resistência à adoção desta prática nas empresas.

Palavras-chave: Capital Humano. Organizações. Desempenho. Vantagem competitiva.

\begin{abstract}
The search for technological innovations is often associated with the increase in the efficiency of machines and equipment, however rarely the human capital of organizations is seen as a source of innovation in the same proportion from physical capital. Even so, the pressure by achieve goals and results is a constant in the everyday lives of the employees of a company. In this context, Coaching place as a practice that seeks to unlock the potential of people to for increase their performance, this article has as main objective to analyze the practice with Coaching as a factor of increasing competitiveness in organizations. As secondary objective, we seek to identify the acceptance of coaching as a recognized and effective method by part companies. For this, the methodology of the article is ruled on a qualitative research, of exploratory and analytical character, and sought through interviews with professionals of the Coaching and with businessmen to reach the proposed objectives. As a result, it has been noted that coaching effectively addresses themes that are often stressful in organizations' day-
\end{abstract}




\section{WWTERHEGETEENOLOGGCA}

to-day and thus causing performance to fall. However, the practice of coaching is still seen as a novelty on the part of the businessmen, who present a certain resistance to the adoption of this practice in companies.

Keywords: Human Capital. Organizations. Competitive advantage.

\section{INTRODUÇÃO}

A inovação tecnológica sempre é colocada como o centro do debate do aumento de competitividade nas organizações. Em um período em que a tecnologia evolui rapidamente, é natural que o foco dos empresários enteja em máquinas mais modernas, novas formas de comunicação ou novos canais de propaganda.

Entretanto, o funcionário também deve ser olhado como um fator fundamental para o aumento da competitividade nas organizações, mais do que as máquinas, o conjunto de funcionários da organização é o principal capital a ser explorado para a evolução da empresa. Além do fato de serem pessoas e não máquinas, os funcionários detém a fonte de sensibilidade e criatividade para a resolução de problemas, portanto, é o capital humano que possui habilidades, conhecimentos, atitudes e competências. Esse capital, se bem administrado, poderá representar o diferencial na organização, e propiciar com que ela obtenha vantagens competitivas.

Neste contexto, aumentar o desempenho do capital humano é uma tarefa complexa. Aumentar o desempenho, neste caso, significa melhorar aspectos da vivência pessoal e profissional do funcionário, como por exemplo, qualidade de vida no trabalho, realizações pessoais, redução do ambiente de stress e melhora na comunicação interna. Para esta tarefa, muitas organizações têm adotado o Coaching. O Coaching é uma prática que estimula o ser humano para que seu potencial aflore e que descubra novos procedimentos de forma natural e espontânea, melhorando o desempenho do seu trabalho na empresa (ARAUJO, 1999).

O objetivo deste artigo é analisar a prática com Coaching como fator de aumento de competitividade nas organizações. Como objetivo secundário, busca-se identificar a aceitação do Coaching como método reconhecido e eficaz por parte das empresas. A importância deste trabalho está no fato de muitas práticas empresariais se configurarem como passageiras ou modismos em um ambiente altamente competitivo, como o das organizações. Será o Coaching realmente eficaz como prática duradoura de aumento de desempenho do capital humano e, por consequência, como fator de aumento da competitividade nas organizações? 


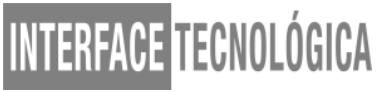

Como metodologia, apresenta-se uma pesquisa qualitativa, com um estudo baseado em entrevistas com profissionais da área de Coaching e empresários de vários setores. A metodologia será detalhada apropriadamente no item 3 deste trabalho, como hipótese de pesquisa, há a percepção de que a valorização do capital humano tende a aumentar a coesão da equipe de uma determinada organização e, assim, fazer com que o ambiente de trabalho seja agradável. Neste ambiente favorável, é possível elevar os níveis exigidos de desempenho da equipe.

\section{O CAPITAL HUMANO}

Contemporaneamente muitas organizações têm visto o investimento em treinamento, capacitação e palestras aos seus colaborados com uma estratégia, para buscar e melhorar o desempenho interno e externo da empresa. No entanto isso não quer dizer que o conhecimento da organização seja os somatórios dos conhecimentos individuais (GODOY, 2008).

Chiavenato (2010, p.10), salienta que "as pessoas devem ser visualizadas como parceiras da organização. Como tais são fornecedoras de conhecimentos, habilidades e competências".

As organizações se conscientizaram de que os funcionários constituem o seu principal ativo, perceberam também que tendem a crescer cada vez mais se forem capazes de aperfeiçoar esses recursos. Colaboradores são capazes de conduzir a organização ao sucesso, estabelecendo parte do seu investimento na expectativa de receber retornos.

Nesse contexto as organizações tratam os colaboradores como parte do seu capital intelectual. Conforme ocorre o aumento da demanda por desenvolvimento de novas competências no ambiente interno das empresas, manifesta- se a imposição de inovações no modo de executar os conteúdos prescritos (BOOG, 2006).

Segundo Chiavenato (2010), existem muitas técnicas de desenvolvimento de pessoas dentro e fora do cargo. Dentro da empresa tem-se a rotação de cargos, posição de assessoria, aprendizagem prática, atribuição de comissões, participações em cursos e seminários externos, exercícios de simulação, treinamento outdoor, estudos de caso, jogos de empresas, centros de desenvolvimento interno e coaching, entretanto fora da empresa inclui a Tutoria ou mentoring e aconselhamento de funcionários. 


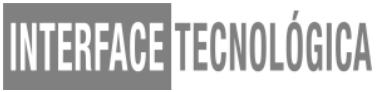

\subsection{A aprendizagem individual}

Segundo Godoy (2008), o capital humano é composto por conhecimento, habilidades, criatividade e experiências individuais dos colaboradores da organização. Consequentemente passam a maior parte do dia dentro das organizações, desta forma aumenta se a possibilidade de desenvolverem diversas habilidades e competências técnicas e/ou humanas.

De acordo com Vygotsky (1988), a aprendizagem é o processo pela qual o indivíduo reúne informações, habilidades, atitudes, valores, abrangendo sempre a interação social, por meio de contato com a realidade, o meio ambiente e as outras pessoas.

Além disso, entendimento das pessoas sobre um determinando contexto ocorre de formas divergentes, ou seja, é necessário entender como cada pessoa aprende. Esses estilos específicos para cada pessoa são desenvolvidos através das combinações de três fatores segundo Godoy (2008, p.228), sendo eles:

- Forma como cada um capta as informações

- Como se organiza e processa essas informações

- Condições necessárias para compreensão e organização dessas informações.

\subsection{Tipos de Aprendizagem}

Aprendizagem experimental de Kurt Lewin que deu amparo para aprendizagem vivencial de Kolb (1997) descreve o conhecimento como um processo de experiências.

Segundo o autor, é necessário o desenvolvimento de quatro tipos diferentes de habilidade pelos aprendizes, para um aprendizado afetivo. Inicialmente, temos a experiência concreta, que são fundamentos que atingem as pessoas no dia a dia para a assimilação de uma informação, posteriormente, refletir sobre essas experiências passadas (observação reflexiva), para assim tirar conclusões da conceptualização dos fatos (conceituação abstrata), por fim usar essas experiências para tomar decisões e resolver problemas (experimentação ativa) conforme (KOLB, 1984).

Em seu modelo de Estilo de Aprendizagem, ele define:

- Conhecimento Acomodativo: Adapta-se sobre circunstâncias imediatas, gosta de estar com pessoas, realizador de coisas, executa plano e experimentos se envolvendo.

- Conhecimento Divergente: Capacidade de imaginação, gerador de ideias, distingue-se pela emoção, interessa-se por pessoas; 


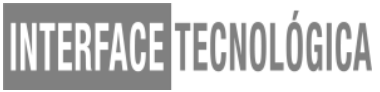

- Conhecimento Assimilativo: Raciocínio indutivo, criação de modelos teóricos, interessa-se por pessoas e preocupa-se com conceitos abstratos;

- Conhecimento Convergente: Destaca-se na aplicação prática de ideias, tem o conhecimento organizado através do raciocínio hipotético-dedutivo, prefere lidar com coisas a pessoas; característico dos engenheiros.

Duas teorias dão suporte aos modelos de aprendizagem o modelo behaviorista e cognitivo. No Modelo Behaviorista o comportamento é observável e mensurável. Já no Modelo Cognitivo- é mais abrangente, pois explica os fenômenos internos. Pode se considerar a aprendizagem como um processo de reunião de conhecimentos, habilidades, conceitos que são armazenados em modelos mentais durante toda a vida podendo mudar ou não o comportamento da pessoa. (GODOY, 2008).

\subsection{Os Modelos de Maslow e Herzberg}

Uma condição preponderante para que o desempenho dos funcionários aumente é a satisfação de suas necessidades, pois quanto mais suas necessidades são atendidas, maior é o resultado para a empresa. Estas necessidades implicam melhores condições de trabalho (AGUIAR, 2005).

A partir da ótica da psicologia, Abraham Maslow (1987) considera que as necessidades humanas apresentam diferentes níveis de classificação. Cada etapa depende de uma condição específica e, a partir desta constatação, o autor conseguiu elaborar uma hierarquia de necessidades, que se classifica em cinco grupos: fisiológicas, de segurança, sociais, de estima e de auto realização. 1). Fisiológicas: São as necessidades mais básicas para a existência e manutenção da sobrevivência da pessoa, como alimentos, sono, vestuário e saneamento. 2) Segurança: refere-se à sensação de proteção e se sentir livre do perigo. Ela tornar-se visível, quando estiver contentada das necessidades fisiológicas. 3). Sociais: estando satisfeita com as necessidades anteriores, se estabelece a necessidade de convívio social e de relacionamento como outras pessoas. 4). Estima: estando satisfeita com a social, ela passará a necessitar de estima, pois ira precisar de reconhecimento dos outros. 5) Auto realização: ao estar satisfeito com a estima, ira necessitar de auto realização que é chegar ao máximo de seu potencial, se tornar aquilo que sempre quis ser (LOPES, 2017). 
Na pirâmide de Maslow, nota-se que as duas primeiras etapas, localizadas na base da pirâmide, são as Necessidades Primárias do ser humano, enquanto as três etapas finais caracterizam as Necessidades Secundárias.

Ao analisar as condições para o ser humano buscar a satisfação de suas necessidades, Herzberg cria a Teoria dos dois fatores. Para Herzberg, a motivação está associada a dois fatores: 1) fatores higiênicos: que diz respeito às condições das pessoas, enquanto trabalha, envolvendo aspectos físicos, salários e políticas da empresa e outros, ou seja, são fatores utilizados pelas empresas para motivar seus funcionários. Quando estes fatores são bem aplicados, eles evitam a insatisfação dos funcionários; e 2) fatores motivacionais: que dizem respeito às necessidades relacionadas ao cargo aumento de produtividade. Neste fator, a motivação tem relação com crescimento e realização. Quando estes fatores são bem utilizados, eles elevam a motivação dos funcionários (LOPES, 2017).

A Ilustração 1 representa a pirâmide das necessidades de Maslow

\section{Ilustração 1 - Pirâmide de necessidades de Maslow}

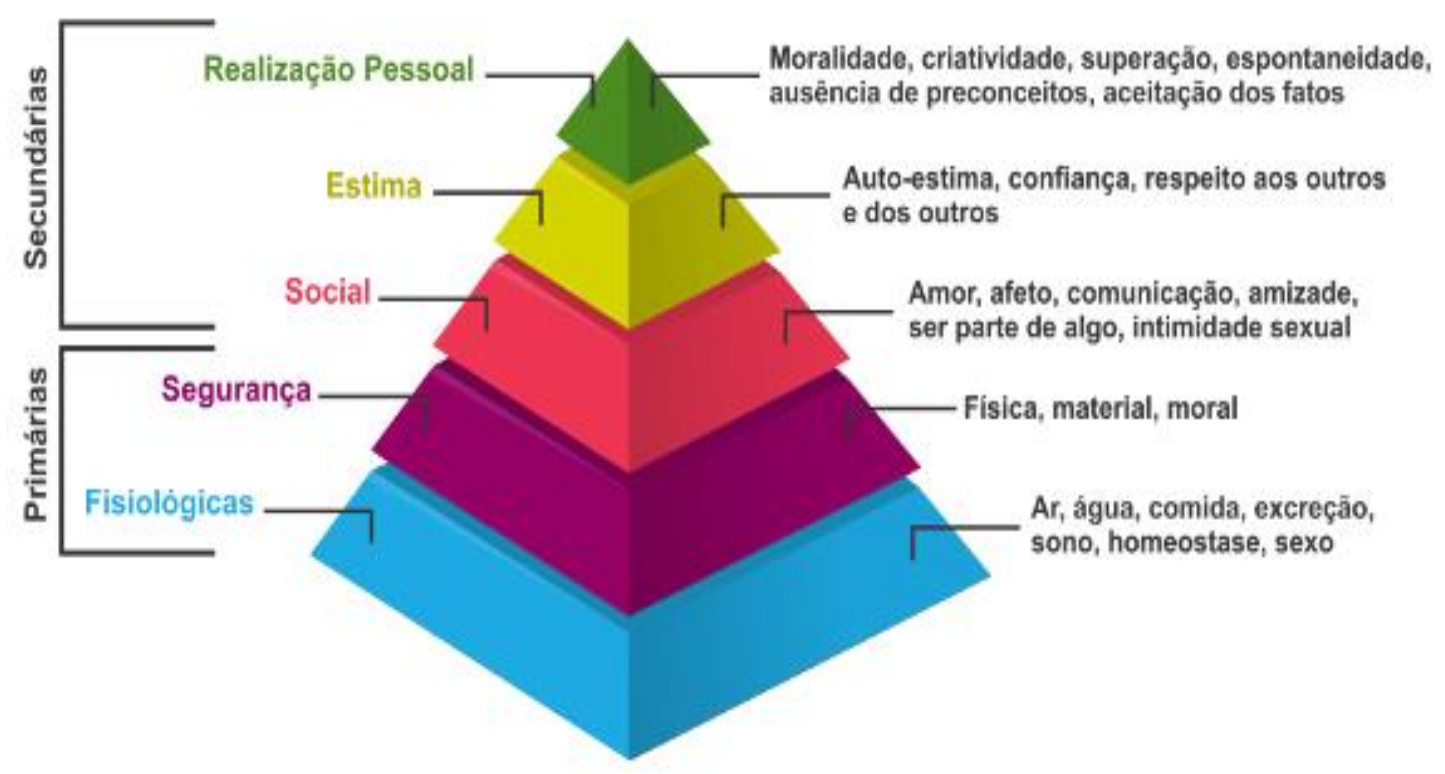

Fonte: Rosemberg (2015) apud Lopes (2017).

A ausência de condições para a satisfação das necessidades ligadas aos fatores higiênicos levaria à desmotivação do funcionário, enquanto a presença dos fatores motivacionais seria um estímulo ao funcionário. A Ilustração 2 representa a Teoria dos dois fatores de Herzberg. 


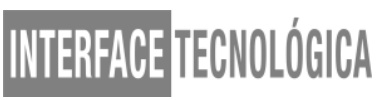

Ilustração 2 - Teoria dos dois fatores de Herzberg

\begin{tabular}{|l|l|}
\hline $\begin{array}{l}\text { Fatores de Motivação } \\
\text { (Os que satisfazem) }\end{array}$ & \multicolumn{1}{c|}{$\begin{array}{c}\text { Fatores de Higiene } \\
\text { (Os que não satisfazem) }\end{array}$} \\
\hline - Realização & - Administração e política da companhia \\
- Reconhecimento & - Supervisão \\
- Responsabilidade & - Relacionamento com supervisor \\
- Avanço & - Condiçōes de trabalho \\
& - Salário \\
& - Relacionamento com colegas \\
& - Vida pessoal \\
& - Relacionamento com os subordinados \\
\hline
\end{tabular}

Fonte: Espínola (2015)

\section{METODOLOGIA}

Como metodologia, este trabalho caracteriza-se por uma pesquisa qualitativa, de caráter exploratório e analítico. As pesquisas exploratórias são usadas quando pouco se conhece o assunto. Assim, o objetivo de uma pesquisa exploratória é compreender um assunto ainda pouco estudado. Este tipo de pesquisa depende da intuição do pesquisador. Por ser um tipo de pesquisa muito específica, quase sempre assume a forma de um estudo de caso (GIL, 2006). Como qualquer pesquisa, ela depende também de uma pesquisa bibliográfica, pois mesmo que existem poucas referências sobre o assunto pesquisado, nenhuma pesquisa hoje começa totalmente do zero.

O estudo de caso, dentro de uma pesquisa exploratória, refere-se a uma situação, entidade ou conjunto de entidades que têm um mesmo comportamento ou são do mesmo perfil. O estudo de caso tem uma profundidade bem maior que os estudos de campo e uma reduzida amplitude em função do baixo número de elementos de pesquisas. Não se podem generalizar as conclusões do estudo de caso, pois são particulares. As conclusões de um estudo de caso geram hipóteses para pesquisas de fenômenos que envolvam um maior contingente de pesquisa (GIL, 2006).

Por fim, a pesquisa explicativa visa identificar os fatores que determinam ou contribuem para a ocorrência dos fenômenos. Segundo Gil (2006), este tipo de pesquisa é a 


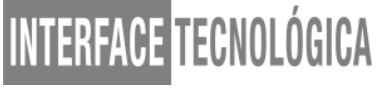

que mais aprofunda o conhecimento da realidade, e, por isso mesmo, está fortemente calcada em métodos experimentais.

Este estudo foi elaborado através de entrevistas com cinco profissionais de Coaching e dez empresários de porte pequeno e médio de diferentes setores de atividade. A entrevista foi realizada sob um roteiro baseado em questionários semiestruturados, que foram analisados de forma qualitativa, na medida em que a opinião dos entrevistados era manifestada de forma livre.

\section{A PRÁtica dO COACHING}

O conceito de Coaching veio do meio esportivo como a designação de um instrutor. O termo coach foi, durante muito tempo, amplamente utilizado em várias modalidades esportivas, especialmente no tênis, no golfe e no esqui. Segundo Whitmore (2012), foi o especialista em tênis pela Harward, Timothy Gallwey que iniciou o trabalho de melhoria de desempenho dos atletas com os livros "The Inner Game os Tennis", “The Inner Skiing” e "The Inner Game of Golf'. A palavra Inner, com a conotação de interior, nos títulos representa o objetivo de trabalhar o estado subjetivo do atleta. Gallwey dizia que o adversário dentro da própria cabeça do atleta seria mais perigoso do que o que estava no time rival. Assim, o Coaching nasce com o objetivo de desbloquear o potencial das pessoas para aumentar o seu desempenho (WHITMORE, 2012).

Ao se criar uma metodologia sistematizada, baseada em aspectos da psicologia e da administração, Gallwey lançou o livro "The Inner Game of Work" que trouxe as práticas do Coaching esportivo para o ambiente do trabalho empresarial. Os objetivos do esporte foram transportados para as metas empresariais de desempenho e o Coaching passou a ser uma prática para aumentar a produtividade das organizações. Assim, o Coaching adquire um caráter inerente à gestão das organizações e deixa para trás aquela conotação esportiva que o conceito tinha em sua origem. Vários autores buscam definir esta adaptação do Coaching à área da gestão das organizações.

Ao ser adaptado para a gestão das organizações, o Coaching passa a abordar aspectos como a motivação do funcionário, a delegação de tarefas, problemas de relacionamento, formação de equipes, desempenho da tarefa, planejamento e revisão e as avaliações internas (WHITMORE, 2012). 


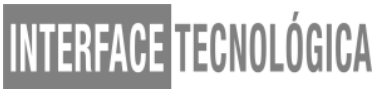

Ilustração 3 - Definição do Coaching por autores selecionados

\begin{tabular}{|c|c|}
\hline Autor & Definição \\
\hline $\begin{array}{l}\text { Parsloe } \\
\text { (1995, p. 18) }\end{array}$ & $\begin{array}{l}\text { "O coaching é diretamente relacionado com o aprimoramento imediato de desempenho } \\
\text { e o desenvolvimento de habilidades, através de uma forma de tutoria ou instrução." }\end{array}$ \\
\hline $\begin{array}{l}\text { Whitmore } \\
\text { (1992, p. 8) }\end{array}$ & $\begin{array}{l}\text { "Coaching é o desbloqueio do potencial de um indivíduo de forma a maximizar seu } \\
\text { desempenho. É ajudar o outro a aprender ao invés de ensiná-lo." }\end{array}$ \\
\hline $\begin{array}{l}\text { Stober, \& Parry } \\
(2005, \text { p. 13) }\end{array}$ & $\begin{array}{l}\text { "Coaching é um processo colaborativo que visa facilitar a habilidade de um cliente } \\
\text { para auto-dirigir seu aprendizado e crescimento, sendo evidenciado por mudanças } \\
\text { significativas na auto-compreensão, auto-conceito e comportamento." }\end{array}$ \\
\hline $\begin{array}{l}\text { Grant } \\
(2005, \text { p. } 4)\end{array}$ & $\begin{array}{l}\text { "Coaching pode ser compreendido como uma metodologia genérica usada para } \\
\text { aprimorar as habilidades e desempenho, assim como o desenvolvimento de individuos. } \\
\text { Ê um processo sistematizado no qual indivíduos são ajudados a explorar seus } \\
\text { problemas, estabelecer metas, desenvolver planos de ação e agir, monitorar e avaliar seu } \\
\text { desempenho de forma a melhor alcançar suas metas [...]." }\end{array}$ \\
\hline $\begin{array}{l}\text { Milaré, \& Yoshida } \\
\text { (2007, p. 88) }\end{array}$ & $\begin{array}{l}\text { "A essência do coaching é ajudar o individuo a resolver seus problemas e a transformar o } \\
\text { que aprendeu em resultados positivos para si e para a equipe a qual lidera." }\end{array}$ \\
\hline $\begin{array}{l}\text { Cox, Bachkirova, \& } \\
\text { Clutterbuck } \\
(2014, \text { p. } 1)\end{array}$ & $\begin{array}{l}\text { "O coaching é um processo de desenvolvimento humano que envolve o uso de } \\
\text { interações estruturadas e focadas e de estratégias, ferramentas e técnicas apropriadas } \\
\text { para promover mudanças desejáveis e sustentáveis, visando o benefício do indivíduo e, } \\
\text { potencialmente, de outros stakeholders." }\end{array}$ \\
\hline
\end{tabular}

Fonte: Oliveira-Silva et al. (2018)

A ilustração 3 representa uma seleção dos principais autores que definem Coaching a partir de pesquisa realizada por Oliveira-Silva et al. (2018).

\section{ANÁLISE DOS DADOS}

Após a realização das entrevistas e a análise das respostas constatou-se que o Coaching atua em fatores que caracterizam as camadas superiores da Pirâmide de Maslow, especialmente na autoestima e na realização moral dos funcionários. Assim, há uma busca inerente por realizações pessoais nestas áreas e o Coaching se coloca como um meio para satisfazer estas necessidades.

$\mathrm{Na}$ medida em que os funcionários, por meio, das práticas do Coaching conseguem gerenciar seus recursos interpessoais e intrapessoais de forma a atingir suas metas, a autoestima vai aumentando gradativamente. Os funcionários passam a enxergar a si mesmos verdadeiramente como Capital Humano das organizações.

Em seguida, com o Capital Humano capaz de lidar com desafios e obstáculos inerentes ao ambiente de trabalho, o Coaching auxilia na formação de lideranças, melhoria do desempenho na função, gestão de carreira e processos de mudança organizacional (OLIVEIRA-SILVA et al. 2018). 


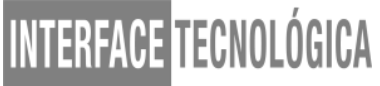

Neste contexto, a melhoria de desempenho do Capital Humano e a conquista de resultados passariam a ser consequências da prática do Coaching. A Ilustração 4 representa as etapas da Pirâmide de Maslow que são o foco principal de atuação do Coaching.

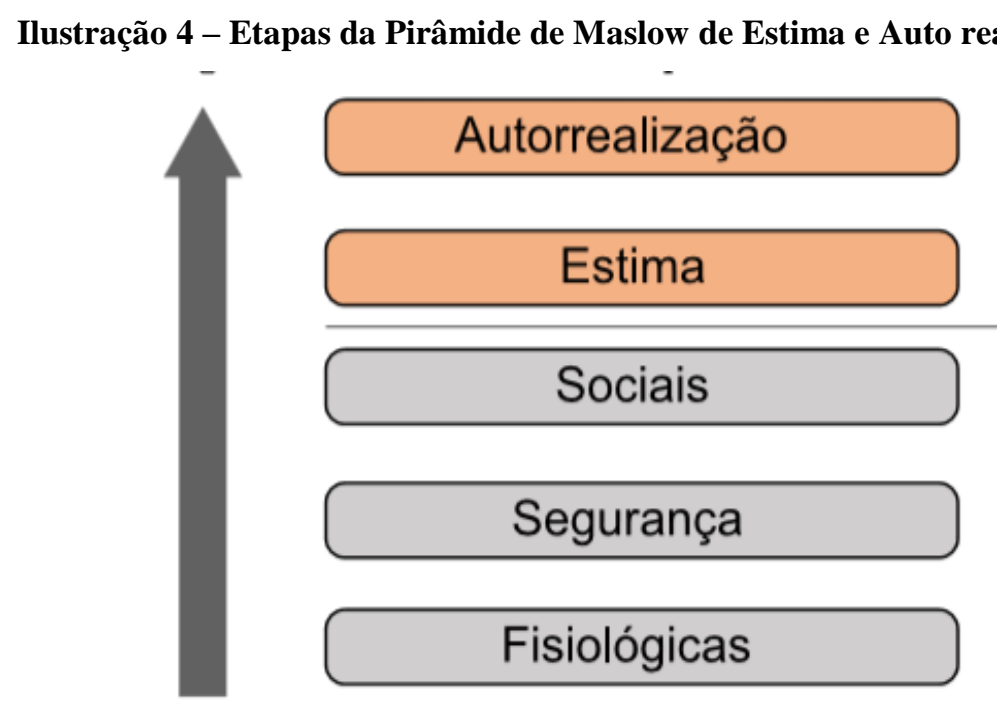

Fonte: Adaptado de SILVA (2008) apud LIMA; AUGUSTO (2018)

De maneira muito similar ao analisado a partir da Pirâmide de Maslow, o Coaching também promove muitos dos os fatores de higiene apresentados na Teoria dos Dois Fatores de Herzberg, como o relacionamento com os colegas, supervisores e subordinados, vida pessoal e ambiente de trabalho.

Esta experiência propicia um empoderamento do Capital Humano que traz consequências positivas para as organizações. O empoderamento abrange, além de reconhecimento, motivação e autonomia; além de senso de participação e vinculo. Criar um contexto adequado ao empowerment significa proporcionar um espaço de trabalho na qual as pessoas estejam incentivadas a buscar suas próprias realizações, a deter informações necessárias, a compreender quais são os resultados, esperados e de que forma a atuação de cada um pode contribuir conforme BOOG (2006, p.98).

Entretanto, os entrevistados afirmaram que ainda falta uma aceitação maior da prática do Coaching por parte dos empresários no Brasil. Esta constatação foi percebida tanto na exposição dos profissionais de Coaching, que afirmam perceber a resistência dos empresários, quanto no posicionamento dos próprios empresários que, em sua maioria, afirmaram não ter interesse em investir no Coaching. 


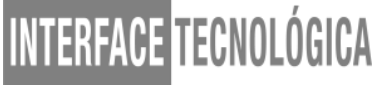

Os empresários entrevistados afirmaram que, apesar de reconheceram a boa iniciativa dos profissionais de Coaching, não estaria disposto a investir nesta prática por não acreditar nas garantias do resultado.

\section{CONCLUSÃO}

As inovações buscadas pelas organizações para aumentar a competitividade no mercado também devem ser aplicadas ao seu capital humano. As organizações devem ver as pessoas como indivíduos dotados de conhecimentos, habilidades, competências e que buscam crescer no ambiente organizacional. Neste contexto, o Coaching se apresenta como uma prática capaz de potencializar o desempenho das pessoas nas organizações.

A partir da revisão de literatura realizada, observou-se que o Coaching atua no topo da Pirâmide de Maslow e trabalha, especialmente, a estima e a auto realização do Capital Humano. Da mesma forma, o Coaching também promove muitos dos os fatores de higiene apresentados na Teoria dos Dois Fatores de Herzberg, como o relacionamento com os colegas, supervisores e subordinados, vida pessoal e ambiente de trabalho.

Entretanto, a adoção da prática do Coaching ainda sofre resistência por parte dos empresários entrevistados, que se mostram incrédulos nos resultados do Coaching de forma duradoura. Assim, resistem em realizar investimentos nesta área.

Superar o desconhecimento dos empresários para, assim, conseguir uma inserção maior no mercado brasileiro é o desafio dos profissionais de Coaching atualmente. Como sugestões de pesquisas futuras, faz-se necessário um acompanhamento da evolução da adoção da prática do Coaching entre os empresários e os motivos que mais sensibilizam os empresários para aceitarem realizar um investimento nesta área.

\section{REFERÊNCIAS}

AGUIAR, M.A.F. Psicologia aplicada a administração. São Paulo. Saraiva, 2005.

ARAÚJO, Ane. Coach: um parceiro para o seu sucesso. São Paulo. Gente, 1999.

BOOG, G.; BOOG, Magdalena. Manual de treinamento e desenvolvimento: processos e operações. São Paulo: Prentice-hall, 2006.

CHIAVENATO, I. Gestão de pessoas. $3^{\text {a }}$ ed. Rio de Janeiro: Elsevier, 2010.

CHIAVENATO, I. Gestão de Pessoas. Rio de Janeiro: Elsevier, 2008. 
CHIAVENATO, I. Gestão de Pessoas: o novo papel dos recursos humanos na organização. 3. ed. Rio de Janeiro: Elzevier, 2011.

CHIAVENATO, I. Introdução à teoria geral da administração. 7. ed.rev. Atual. Rio de Janeiro: Elsevier, 2004.

ESPÍNOLA, H. L. O Salário: Pode Ser Usado Como um Fator Motivador? Disponível em https://psicologado.com.br/atuacao/psicologia-organizacional/o-salario-pode-ser-usado-comoum-fator-motivador Acesso em 25/09/2018.

GIL, Antônio Carlos. Métodos e técnicas de pesquisa social. São Paulo: Editora Atlas 2006

GODOY, Arilda Shmidt (et al.). Gestão do fator humano: Uma visão baseada em stakeholders. 2. ed. São Paulo: Saraiva 2008.

KOLB, D.A. A gestão e o processo de aprendizagem. In: STARKEY, K. Como as organizações aprendem. São Paulo: Futura, 1997. P.321-341.

KOLB, D.A. Experimental learning: Experience as the source of learning and Development. New Jersey: Prentice-Hall. 1984

LIMA, A. S.; AUGUSTO, C. A. O impacto das práticas de gestão de pessoas sobre a motivação do colaborador em organizações da sociedade civil de interesse público (OSCIPS). Rev. Ciênc. Empresa. UNIPAR, Umuarama, v. 19, n. 1, p. 17-40, jan. /jun.2018.

LOPES, M. R.; OLIVEIRA, W. N. P: QUEIRÓS, A. F. Motivação e Liderança Dentro das Empresas. Rev. Cienc. Gerenc., v. 21, n. 34, p. 91-97, 2017

MARRAS, J. P. Administração de recursos humanos - do operacional ao estratégico. São Paulo: Editora Saraiva 2011.

MARRAS, J. P. Administração de recursos humanos: do operacional ao estratégico. 14. ed. São Paulo: Saraiva 2011.

OLIVEIRA-SILVA, L. C. et al. Desvendando o Coaching: uma Revisão sob a Ótica da Psicologia. Psicol. Cienc. prof., Brasília, v. 38, n. 2, p. 363-377, jun. 2018. Disponível em <http://www.scielo.br/scielo.php?script=sci_arttext\&pid=S1414-

98932018000200363\&lng=pt\&nrm=iso>. Acessos em 02 out. 2018.

RESENDE, E. (2000). O livro das competências: desenvolvimento das competências: a melhor auto-ajuda para pessoas, organizações e sociedade. Rio de Janeiro: Qualitymark.

RUAS (2000) FLEURY; FLEURY (2001) e DUTRA, J. S. Gestão de pessoas: modelo, processos, tendências e perspectivas. São Paulo: Atlas, 2002.

RUAS, R. L. (2001). Desenvolvimento de Competências Gerenciais e a Contribuição da Aprendizagem Organizacional. In M. T. Fleury, \& M. Oliveira (Orgs.), Gestão Estratégica do Conhecimento. São Paulo: Atlas. 
VYGOTSKY, L.S. A Formação Social da Mente. $6^{\text {a }}$ ed. São Paulo, SP. Martins Fontes Editora LTDA, 1998.

VYGOTSKY, L.S; LURIA, A.R. \& LEONTIEV, A.N. Linguagem, desenvolvimento e aprendizagem. São Paulo: Ícone: Editora da Universidade de São Paulo, 199.

WHITMORE, J. Coaching para aprimorar o desempenho: Os princípios e as práticas do coaching e da liderança. Clio Editora. São Paulo, 2012. 